\title{
Open Mass Cultures of Marine Microalgae for Biodiesel Production: Laboratory Approach to Study Species Competition in Mixed Cultures
}

\author{
Marina Mingazzini, Maria Teresa Palumbo \\ Water Research Institute, IRSA, CNR, Brugherio, Italy \\ Email: mingazzini@irsa.cnr.it
}

Received 19 February 2015; accepted 8 March 2015; published 12 March 2015

Copyright (C) 2015 by authors and Scientific Research Publishing Inc.

This work is licensed under the Creative Commons Attribution International License (CC BY). http://creativecommons.org/licenses/by/4.0/

(c) $\underset{\mathrm{EY}}{\mathrm{O}}$ Open Access

\begin{abstract}
Since the interspecific competition represents a critical issue for biodiesel production in open mass cultures of microalgae, laboratory studies aimed to select competitive species receive significant interest. In this work, a laboratory approach based on the method of multisizing cell counting was developed to monitor the competition course of single algal species grown in mixed cultures. Two marine microalgae, Dunaliella tertiolecta and Phaeodactylum tricornutum, were used to setup an induced-contamination assay, with the aim to test their competitive capabilities. The evaluation of the oil production by dried biomass extraction was coupled to the growth assay to investigate the effect of competition on oil yield. The adopted experimental approach revealed effective as a method to selectively measure the algal growth of single species in mixed cultures, enabling to evaluate the competitive properties of Dunaliella outgrowing the contaminant species Phaeodactylum. At the end of the experiment the dominant species contributed more than $90 \%$ to the total biomass, while no loss of oil production was observed, the oil yield in the mixed being even higher than in the unialgal culture ( $3.15 \mathrm{vs} .2 .28 \mathrm{mg}$ ). The outcomes of the induced competition suggest the use of Dunaliella as a competitive oil-producer species and especially support the potential of the experimental approach to be used for preliminary screening to drive species selection for open mass cultures.
\end{abstract}

\section{Keywords}

Biodiesel, Marine Microalgae, Interspecific Competition, Multispecies Algal Assay, Oil-Yield 


\section{Introduction}

Microalgae biomass is currently considered the most promising alternative as oil source for biodiesel production [1] [2]. When compared to the conventional oil-crops, microalgae, as the most efficient primary producers of biomass, appear to be the only source of renewable biodiesel that is capable of meeting the global demand for transport fuels. However, the commercial production of algal biodiesel is yet to become reality [2] [3]. For commercial oil production the algae should be cultured on a large scale at high productivity in open-pond systems, which can be economically feasible since mainly based on the natural solar energy [4]. Such cultivation systems, when supplied by natural sea-water, have also the additional advantage to potentially decrease up to $90 \%$ water requirement. Further, the use of brackish microalgae as feedstock allows increasing the inflow of fresh wastewater as nutrient source, providing the additional benefit of water treatment [5].

A key drawback of the outdoor systems is that, due to direct exposure to the atmosphere, the open mass cultures revealed susceptible to contamination by autochthonous undesired microalgae which can outcompete the inoculated strains [3] [4] [6]. Currently, monoculture mass-cultivation is achieved by maintenance of extreme environment conditions (e.g. high salinity, high $\mathrm{pH}$ ) which could exclude competing species. Unfortunately, such conditions revealed inappropriate to maximize the overall production of most of oleaginous species [6] [7].

The early studies on biodiesel production from microalgae, undertaken over the past few decades under the Aquatic Species Program [8] were mostly devoted to the selection of species with high oil content, but it was more recently found that the most oil rich species generally present low competition properties, mostly due to a low rate of growth [9]. It is currently suggested that, in addition to the specific oil content, the competitive advantage should be tested before selecting species and setting up mass culture systems [10]. While wide research has been carrying out on how to manage contamination and to understand the role of varying environmental factors, laboratory studies on interspecific competition still represent a basic research need aimed to avoid the risk of lowering the overall productivity likely associated to the contamination event in open pond systems [10] [11]. In this work a laboratory approach was developed with the aim to test the competitive capabilities of two marine microalgae, Dunaliella tertiolecta and Phaeodactylum tricornutum, by induced contamination bioassay. The difference of Mean Cell Volume (MCV) of the selected species was just used to discriminate the two sizedistributions by means of a multi-channel particle counter as a strategy for a simultaneous counting and sizing of single species growing in mixed cultures. The effect of the induced competition on the overall productivity was evaluated by coupling the measurement of the oil yield to the cell growth in the cultures.

\section{Material and Methods}

\subsection{Species Selection}

The marine species Dunaliella tertiolecta and Phaeodactylum tricornutum have been maintained in our laboratory according to EPA standard procedures [12]. The two species have similar nutritional requirements, similar specific dry weight, and both are characterized by high tolerance within a large range of salinity [13] [14]. Hence, they seem just appropriate to perform a competition experiment.

While both algae are oleaginous species, the oil content on biomass dry weight of Dunaliella is reported in a range of values (20\% - 63\%) higher than Phaeodactylum (20\% - 30\%) [1] [3] [14]-[16]. Dunaliella, which is a motile species of green algae particularly easy to cultivate, is recommended as "promising" species for mass cultures [14]. By contrast, the diatom Phaeodactylum, which is a typically fast-growing alga, is reported as "undesired" contaminant species in mass culturing of marine microalgae, mainly because of its low-food value for aquaculture applications [17].

\subsection{Induced Competition Assay}

Based on the above described characteristics of the selected species, Phaeodactylum was used to contaminate Dunaliella. The interspecific competition was induced by the experimental addition of exponentially growing cells of Phaeodactylum (2200 cells/ml) to the unialgal culture of Dunaliella at the incubation day 2. Phaeodactylum cells were added by a ratio of $1: 20$, just to simulate a contamination event. The mixed culture was paralleled by unialgal control cultures of each species. All culture flasks were incubated under the same condition of $20^{\circ} \mathrm{C} \pm 1^{\circ} \mathrm{C}, 14 \mathrm{~h}: 10 \mathrm{~h}$ light:dark, cool-white fluorescent tubes (5000 lux), manual shake twice a day. The algal growth, as cell density (cells/ml), the biomass volume $\left(\mu \mathrm{m}^{3} / \mathrm{ml}\right)$ and the growth rates, as doublings per day 
(d/day), were measured until the stationary phase was reached in all the incubated cultures.

\subsection{Specific Algal Counting}

The algal growth measurement was performed by using the electronic particle counter (mod. Beckman Coulter Multisizer $^{\mathrm{TM}}$ 3). The technique is based on the Coulter Principle, also known as Electrical Sensing Zone method, allowing particles homogeneously suspended in a conducting solution to be simultaneously counted and sized. The counting system is thus able to accurately measure the cell number, as electrical pulses, as well as the volume of each drawn cell, within a wide size range. Aimed to define the specific size ranges of Phaeodactylum and Dunaliella a large number of preliminary analyses were performed on single cultures. After that, the analytical range appropriate to cover the two different size distributions was defined and used to perform the cell count of all cultures. The selected range $10-300 \mu \mathrm{m}^{3}$ was adopted since just enabling to discriminate the growth of the single species in mixed cultures.

\subsection{Oil Extraction}

The final biomass produced by contaminated and uncontaminated cultures was simultaneously harvested by centrifugation at the end of the experiment, hence dried in a thermostatic oven at $70^{\circ} \mathrm{C}$. The dry weight of biomass was gravimetrically measured and expressed as milligrams. The oil content was solvent-extracted from the dried biomass by homogenization in propan-2-ol:cycloexane:water 8:10:11 by volume [18]. Samples were exposed to 20 minutes sonication to maximize the extraction of intracellular oil. After separation of the organic phases by centrifugation, the oil was collected from the upper layer and gravimetrically measured after drying for 1 hour at $103^{\circ} \mathrm{C}$. The cell oil content was expressed as percent of dry biomass weight.

\section{Results and Discussion}

The results of the preliminary analyses of unialgal cultures of Phaeodactylum and Dunaliella performed by multichannel counting to define their specific size ranges are shown in Figure 1.

Due to the Digital Pulse Processing technology of the instrument (Beckman Coulter Multisizer ${ }^{\mathrm{TM}} 3$ ), two distinct size-distributions, each one divided into 300 size channels, have been clearly defined for the two species, the MCV being $72 \mu^{3}$ and $166 \mu^{3}$, for Phaeodactylum and Dunaliella, respectively. As a result, we could select narrow ranges of interest respectively below and above $107 \mathrm{~mm}^{3}$ to discriminate the growth of the two single species when mixed in the competition experiment cultures.

The evolution trend of both species in the cultures was studied over 19 incubation days of competition assay. In Figure 2 the growth curves of Phaeodactylum and Dunaliella, simultaneously monitored by cell-counting in the mixed and in the unialgal controls, are compared.

First of all, the fact that the Multisizer counting revealed able to simultaneously count the single species until the end of the experiment is to be appreciated, when considering that in previous investigations using haemocytometer counting method to study competition, most of the experiments failed before the outcome of competition, even between the same species, just because of difficulties in counting individual species, mainly due to cell clumping [17].

The growth curve of Dunaliella in the mixed was similar to that of the control culture, the stationary phase reaching maximum cell densities of 2 and $2.2 \times 10^{6}$ cells/ml respectively, after two weeks of incubation. By contrast, the growth of Phaeodactylum was markedly different when comparing the control with the mixed culture, where the contaminant species, which was inoculated at the incubation day 2, stopped growing in few days, reaching stationary values of $160 \times 10^{3}$ cells/ml at the experiment day 6 , revealing unable to compete against Dunaliella, which successfully kept growing like its control, reaching the stationary phase at the incubation day 13. It should be noted that, even if all the cultures were grown under the same nutrient conditions, the cell density achieved by Phaeodactylum in the control culture $\left(4.4 \times 10^{6}\right.$ cells $\left./ \mathrm{ml}\right)$ at the end of the experiment was two-fold higher than Dunaliella in control or mixed cultures. This large difference observed on cell number can be definitely explained by the specific difference of cell volume, Phaeodactylum MCV being two-fold lower than Dunaliella MCV. Actually, when considering the biomass volume, as measured by the particle counter, the much similar values of $350 \times 10^{6}$ vs. $320 \times 10^{6} \mathrm{\mu m}^{3} / \mathrm{ml}$ were respectively found in Dunaliella and Phaeodactylum single cultures. 


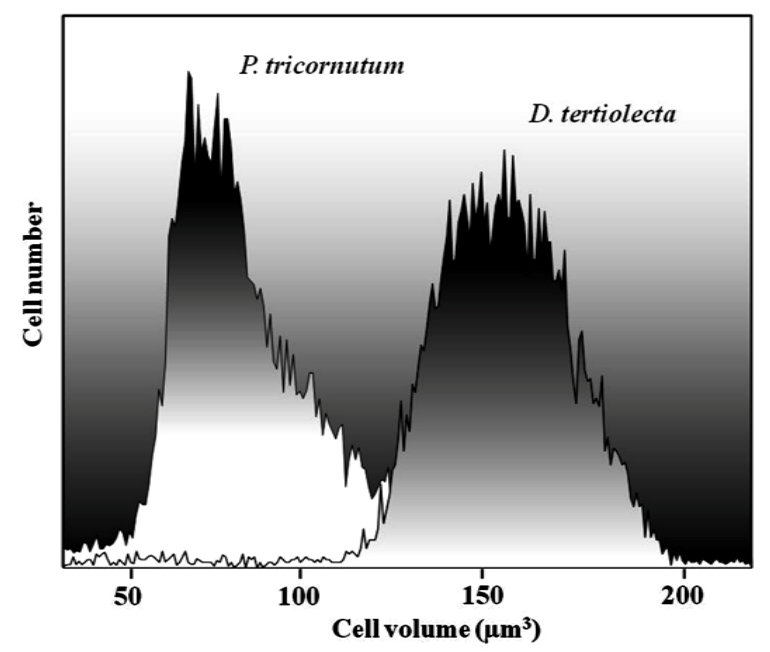

Figure 1. Size distribution of Phaeodactylum and Dunaliella as determined by the multichannel particle counting.

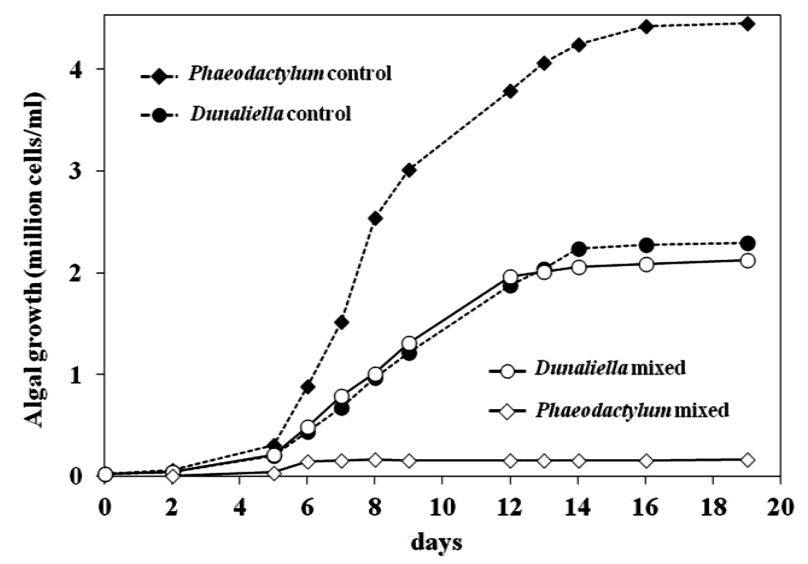

Figure 2. Phaeodactylum and Dunaliella cell growth in the mixed (solid line) and in the control (dashed line) cultures.

The invasive capacity of the contaminant species Phaeodactylum is revealed only when comparing the growth rates of individual species, as measured in the contaminated culture. Figure 3 shows the competitive properties of Phaeodactylum during the first days of growth until day 6. In particular, while for the chlorophyte Dunaliella a maximum growth rate of $1.23 \mathrm{~d}$ /day was measured in the mixed culture, the diatom Phaeodactylum exhibited the highest rate of growth of $1.93 \mathrm{~d}$ /day, which was even greater than $1.52 \mathrm{~d} / \mathrm{day}$, measured in its unialgal control.

The drop in cell doubling shown after the experimental day 6 can likely be due to the limitation of nutrient resources. According to the distinctive properties of the species, as reported by previous studies [14], the main reason of the success of Dunaliella against the fast growing diatom is likely to be explained by its high efficiency in the uptake of available nutrient resources, which permitted holding dominant position in the competition. The evolution trend of competition is just clear in Figure 4, where the percent contribution of single species to the mixed culture is shown.

The data obtained by means of the counting and sizing method were used to calculate the percent contribution of single species to the total biomass in the mixed culture, as plotted in Figure 4. It is to be noted that, due to the large difference in cell volume of the two species, the specific percent values are significantly different depending on whether cell number or biomass volume is considered.

The increasing trend of Phaeodactylum reached the maximum contribution of $23 \%$ to the total cell number versus $12 \%$ of biomass at day 6 . While the contribution of Dunaliella was decreasing from $100 \%$ to $77 \%$ of the 


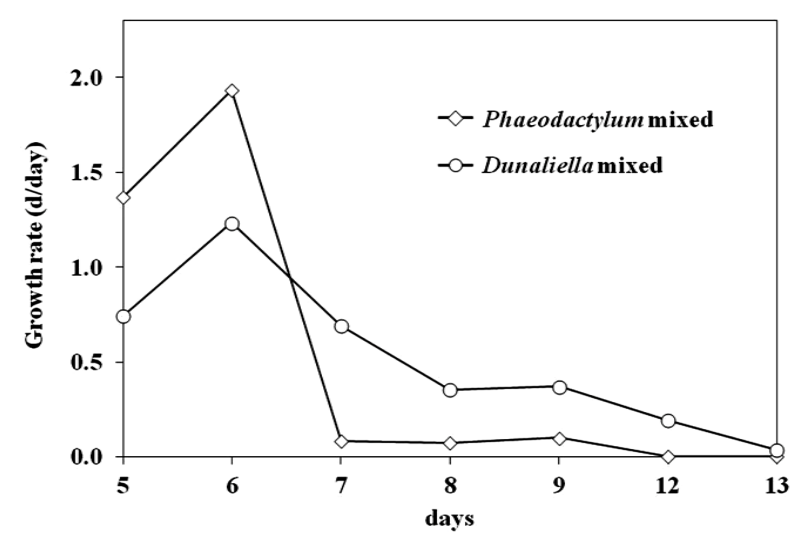

Figure 3. Comparison of the specific growth rates (d/day) during contamination.

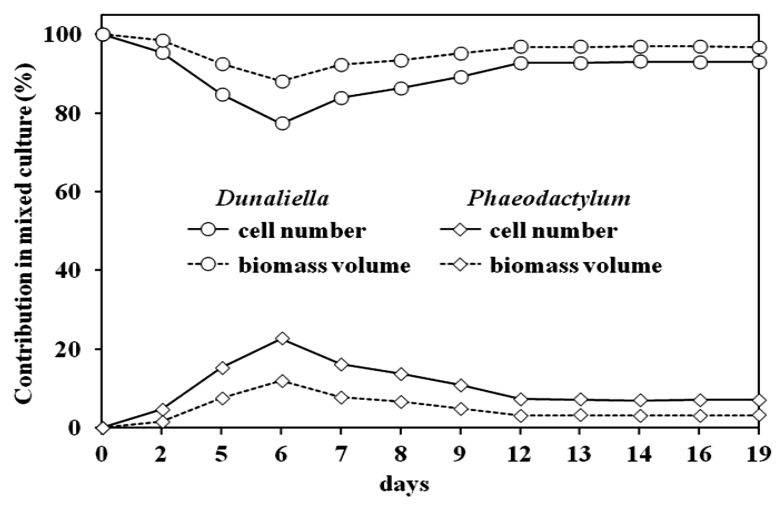

Figure 4. Percent contribution in cell number and in biomass volume of the two species in the mixed culture.

total cell number within 6 days, the chlorophyte progressively recovered its position of absolute dominance, with a final contribution up to $93 \%$ of total cells, and up to $97 \%$ of total biomass, at the end of the experiment, that is when the biomass was harvested, and the oil extracted, from all the cultures. In the case of the mixed culture the percent contribution of total yield was used to calculate the biomass and the oil yield produced by individual species. All the values of biomass and oil yield are shown in Table 1, where control and mixed cultures are compared.

As expected on the basis of the trophic potential of the culture medium, similar values of total biomass yield were measured in the cultures, in the mixed being even slightly higher than in the uncontaminated control, where Dunaliella was growing alone. In particular, the reliability of the multisizing counting method is highlighted when considering that similar values of biomass of the unialgal cultures of Dunaliella and Phaeodactylum, in spite of their great differences in cell number, were found either when gravimetrically measured as biomass yield, or by electronic counting as biomass volume. Furthermore, against the large difference found for Phaeodactylum when comparing individual species in the mixed with their unialgal controls, the much similar values of 8.83 and $8.59 \mathrm{mg}$ found for Dunaliella confirm its undisturbed and absolute dominance in the competition and mainly support the capacity of the adopted counting technique to discriminate the contribution of single algal species to the total biomass in mixed cultures.

Likewise, very close values of oil yield were found in the unialgal cultures of Dunaliella and Phaeodactylum, while the oil production in the mixed culture was not lowered by the induced competition, being instead higher than in the uncontaminated control culture. The oil content, calculated as percent oil of the biomass yield, revealed a good agreement of all the specific oil production with literature data. Apart from the oil contribution of Phaeodactylum in the mixed culture $(0.1 \mathrm{mg})$, which was regarded as negligible, the value of $26.16 \%$ of biomass, calculated in its unialgal control is just falling in the middle of the $20 \%-30 \%$ range reported by the literature [1] 
Table 1. Biomass and oil yields measured in the unialgal controls and in the mixed culture.

\begin{tabular}{ccccc}
\hline Culture & Species & Biomass (mg) & Oil yield (mg) & Oil content (\%) \\
\hline \multirow{2}{*}{ Unialgal } & Dunaliella & 8.59 & 2.28 & 26.54 \\
& Phaeodactylum & 7.99 & 2.09 & 26.16 \\
\multirow{2}{*}{ Mixed } & Dunaliella & 8.83 & 3.05 & 34.54 \\
\hline
\end{tabular}

[3]. Likewise the $26.54 \%$ oil of Dunaliella control is consistent with the $20 \%$ - $63 \%$ literature range [14]-[16]. Also, if considering the $34.54 \%$ oil as likely corresponding to oil content of Dunaliella in the mixed culture, that value would overlap the average of the same specific range.

In any case, instead of a loss of the overall productivity, the obtained results have rather pointed out the increase of oil production in the mixed culture, as an effect of the induced competition. It seems in agreement with recent literature supporting that the enhancement of the oil production can be linked to the microalgal species diversity of mixed cultures. The same authors are even suggesting that multispecies algal communities, instead of single species, should be cultivated in open-pond mass cultures to store more energy as lipids for biofuel production [11] [19].

\section{Conclusions}

The adopted laboratory approach coupling the experimental design of induced competition with the instrumental multisizer-counting method proved effective showing high efficiency of Dunaliella, which exhibited competitive abilities to resist to the contamination of Phaeodactylum and to produce as high biomass in mixed as in unialgal culture condition. Also the oil production, which was not affected by the contamination event, supported the suitability of Dunaliella to be used in open mass-cultures for biodiesel production.

The use of the multichannel analyzer proved to be a sensitive and reliable method, capable to discriminate between two single species growing in mixed cultures, even at high cell-density levels, thus enabling to follow the evolution trend of competition as well as the calculation of the specific contribution to the total, biomass and oil yield, in mixed cultures. The obtained results strongly supported the adopted counting method as an effective strategy to study the interspecific competition between oil-producer algal species.

The adopted experimental approach supports the potential of laboratory algal assays for preliminary screening to drive species selection, in terms of their competitiveness, for their potential use in outdoor systems. The use of this approach is recommended as useful means to prevent the risk of lowering the oil yield which is possibly associated to the interspecific contamination in open mass-cultures for biodiesel production.

\section{References}

[1] Demirbas, A. and Demirbas, M.F. (2011) Importance of Algae Oil as Source of Biodiesel. Energy Conversion Management, 52, 163-170. http://dx.doi.org/10.1016/j.enconman.2010.06.055

[2] Chisti, Y. (2013) Constraints to Commercialization of Algal Fuels. Journal of Biotechnology, 167, 201-214. http://dx.doi.org/10.1016/j.jbiotec.2013.07.020

[3] Chisti, Y. (2007) Biodiesel from Microalgae. Biotechnology Advances, 25, 294-306. http://dx.doi.org/10.1016/j.biotechadv.2007.02.001

[4] Schenk, P.M., Thomas-Hall, S.R., Stephens, E., Marx, U.C., Mussgnug, J.H., Posten, C., Kruse, O. and Hankamer, B. (2008) Second Generation Biofuels: High-Efficiency Microalgae for Biodiesel Production. Bioenergy Resource, 1, 2043. http://dx.doi.org/10.1007/s12155-008-9008-8

[5] Yang, J., Xu, M., Zhang, Z., Hu, Q., Sommerfeld, M. and Chen, Y. (2011) Lyfe Cycle Analysis on Biodiesel Production from Microalgae: Water Footprint and Nutrients Balance. Bioresource Technology, 102, 159-165. http://dx.doi.org/10.1016/j.biortech.2010.07.017

[6] Vasudevan, P.T. and Briggs, M. (2008) Biodiesel Production-Current State of the Art and Challenges. Journal of Industrial Microbiology and Biotechnology, 35, 421-430. http://dx.doi.org/10.1007/s10295-008-0312-2 
[7] Stephens, E., Ross, I.L., Mussgnug, J.H., Wagner, L.D., Borowitzka, M.A., Posten, C., Kruse, O. and Hankamer, B. (2010) Future Prospects of Microalgal Biofuel Production Systems. Trends in Plant Science, 15, 554-564. http://dx.doi.org/10.1016/j.tplants.2010.06.003

[8] Sheehan, J., Dunahay, T., Benemann, J. and Roessler, P. (1998) A Look Back at the US Department of Energy's Aquatic Species Program: Biodiesel from Algae. Close-Out Report. NREL/TP-580-24190, NREL, DOE, Golden. http://dx.doi.org/10.2172/15003040

[9] Mingazzini, M., Palumbo, M.T. and Pagnotta, R. (2008) Oil-Production by Microalgae: An Interspecific Comparison. Proceedings of the 1st International Symposium on Green Chemistry, Munich, 13-16 October 2008, 85-87.

[10] Wang, H., Zhang, W., Chen, L., Wang, J. and Liu, T. (2013) The Contamination and Control of Biological Pollutants in Mass Cultivation of Microalgae. Bioresource Technology, 128, 745-750. http://dx.doi.org/10.1016/j.biortech.2012.10.158

[11] Stockenreiter, M., Graber, A.K., Haupt, F. and Stibor, H. (2012) The Effect of Species Diversity on Lipid Production by Micro-Algal Communities. Journal of Applied Phycology, 24, 45-54. http://dx.doi.org/10.1007/s10811-010-9644-1

[12] EPA (1974) Marine Algal Assay Procedure: Bottle Test. US Environmental Protection Agency, Corvallis.

[13] Mingazzini, M. and Palumbo, M.T. (2008) Proposal of Test Species Useful to Evaluate the Algal Growth Potential (AGP) in Estuarine Mixing Area. Biologia Marina Mediterranea, 15, 390-391.

[14] Hosseini Tafreshi, A. and Shariati, M. (2009) Dunaliella Biotechnology: Methods and Applications. Journal of Applied Microbiology, 107, 14-35. http://dx.doi.org/10.1111/j.1365-2672.2009.04153.x

[15] Minowa, T., Yokoyama, S., Kishimoto, M. and Okakura, T. (1995) Oil Production from Algal Cells of Dunaliella tertiolecta by Direct Thermochemical Liquefaction. Fuel, 74, 1735-1738. http://dx.doi.org/10.1016/0016-2361(95)80001-X

[16] Takagi, M., Karseno and Toshiomi, Y. (2006) Effect of Salt Concentration on Intracellular Accumulation of Lipids and Triacylglyceride in Marine Microalgae Dunaliella Cells. Journal of Bioscience and Bioengineering, 101, 223-226. http://dx.doi.org/10.1263/jbb.101.223

[17] Goldman, J.C., Riley, C.B. and Dennett, M.R. (1982) The Effect of pH in Intensive Microalgal Cultures: Species Competition. Journal of Experimental Marine Biology and Ecology, 57, 15-24. http://dx.doi.org/10.1016/0022-0981(82)90141-1

[18] Smedes, F. (1999) Determination of Total Lipid Using Non-Chlorinated Solvents. The Analyst, 124, 1711-1718. http://dx.doi.org/10.1039/a905904k

[19] Smith, V.H. and Crews, T. (2014) Applying Ecological Principles of Crop Cultivation in Large-Scale Algal Biomass Production. Algal Research, 4, 23-34. http://dx.doi.org/10.1016/j.algal.2013.11.005 